\title{
A TEORIA SENTIDO-TEXTO E SUAS POSSIBILIDADES DE APLICAÇÃO EM DICIONÁRIOS DE APRENDIZES DO ESPANHOL
}

\section{MEANING-TEXT THEORY AND ITS POSSIBILITIES OF APPLICATION TO SPANISH LEARNER'S DICTIONARIES}

Laura Campos de Borba

Universidade Federal do Rio Grande do Sul Porto Alegre, Rio Grande do Sul, Brasil

RESUMO: Estudantes de línguas estrangeiras precisam de ferramentas linguísticas confiáveis que descrevam a língua em uso, por um lado, e que satisfaçam as suas necessidades, por outro lado. Dicionários de aprendizes são ferramentas compiladas para auxiliar esse perfil de usuário em tarefas de compreensão e de produção. As colocações formam parte do conjunto dos principais fenômenos atinentes à produção. Esse tipo de combinatória carece de uma melhor descrição em muitos dicionários de aprendizes, especialmente nos dicionários de aprendizes de espanhol (DAEs) devido ao seu tratamento insatisfatório. $\mathrm{O}$ objetivo deste artigo é explorar a possibilidade de aplicação da Teoria SentidoTexto (TST) para descrever colocações em DAEs. Primeiramente, introduzimos os postulados gerais da TST. Em seguida, listamos e analisamos colocações incluídas em DAEs e dicionários monolíngues de espanhol, comparando-as com dados de corpus. Por fim, aplicamos a TST na descrição das colocações encontradas e propomos uma aplicação da formalização da TST. Conclui-se que a TST oferece um método bastante útil para descrever colocações. A próxima etapa desta pesquisa é apresentar as informações linguísticas de maneira ainda mais amigável para o consulente dos DAEs.

PALAVRAS-CHAVE: Lexicografia Pedagógica; Dicionários de aprendizes de espanhol; Teoria SentidoTexto; Colocações; Aplicação das funções lexicais.

ABSTRACT: Foreign language learners need reliable language tools that could describe language in use on the one hand and fulfill their needs on the other hand. Learner's dictionaries are tools compiled to assist this user profile on understanding and producing language. 
Collocations figure in the set of major linguistic phenomena related to production tasks. This type of combinatory pattern demands better descriptions in many learner's dictionaries, but particularly on Spanish learner's dictionaries (SLDs) due to their poor and bad description. This paper aims to explore the possibility to employ the Meaning-Text Theory (MTT) to describe collocations in SLDs. First, we introduce general MTT postulates. Secondly, we list and analyze collocations presented by SLDs and monolingual Spanish dictionaries and compare this information with corpus data. Finally, we apply MTT to describe the set of collocations founded and propose an application of the lexical functions' formalization. In conclusion, MTT offers a very useful method to describe collocations. The next step of this research is to present linguistic data in an even friendly way for the learner.

KEYWORDS: Pedagogical Lexicography; Spanish learner's dictionaries; Meaning-Text Theory; collocations; lexical functions' application. 


\section{INTRODUÇÃO}

Estudantes de línguas estrangeiras precisam de ferramentas linguísticas confiáveis que descrevam a língua em uso, por um lado, e que satisfaçam as suas necessidades, por outro lado. Dicionários de aprendizes são ferramentas compiladas para auxiliar esse perfil de usuário em tarefas de compreensão e de produção. O espanhol, por exemplo, possui três expoentes dessa classe de obras: o Diccionario Señas para la enseñanza de la lengua española para brasileños (SEÑAS, 2001), o Diccionario de la Lengua Española para Estudiantes de Español (DiELE, 2002) e o Diccionario Salamanca de la Lengua Española (DiSal, 2006). Cabe observar, entretanto, que ditas obras oferecem uma descrição deficitária da língua, seja pela falta de informações da língua em uso, seja pela inclusão de informações que não encontram respaldo em corpora (BORBA, 2017).

Um dos fenômenos atinentes à produção são as colocações. Apesar de almejarem auxiliar seus consulentes em tarefas dessa natureza, os dicionários supracitados raras vezes incluem colocações nos seus verbetes; quando isso ocorre, são poucos os casos descritos .

O objetivo do presente trabalho é explorar as possibilidades de aplicação da Teoria Sentido-Texto para a descrição de colocações em dicionários de aprendizes de espanhol.

\section{A TEORIA SENTIDO-TEXTO (TST)}

Conforme Mel'čuk (1997, p. 4-6), há três postulados gerais que caracterizam a TST em linhas gerais. Primeiramente, o autor define língua como um sistema finito de regras (sintáticas e morfológicas) que intermediam a correspondência entre infinitos sentidos (nível semântico) e infinitos textos (realizações da língua). Segundo a TST, um sentido pode manifestar-se por meio de distintos textos, enquanto um mesmo texto pode corresponder a diversos sentidos. Em segundo lugar, a TST é uma proposta de descrição exaustiva de uma unidade léxica (doravante UL) em todos os seus níveis de representação (semântico, sintático, morfológico e fonológico). Para os adeptos da mesma, descrever uma UL consiste em descrever todas as suas possibilidades de realização (textos). Em terceiro lugar, o nível semântico é central para a descrição, enquanto os níveis sintático e morfológico são importantes para a investigação da TST justamente por sua tarefa de intermediar sentidos e textos.

Consoante o primeiro postulado geral da TST, há duas vias de correspondência entre sentidos e textos: a análise, que corresponde à compreensão da fala e que parte de um texto para o(s) seu(s) sentido(s); e a síntese, que corresponde ao falar e que parte de um sentido para o(s) seu(s) texto(s) correspondentes. Entretanto, Mel'čuk e Milicévic (2014, p. 78-79) defendem a perspectiva da síntese por três razões. Em primeiro lugar, e sob o ponto de vista "leigo", é muito mais comum afirmar que um indivíduo fala (ou 
não) uma determinada língua (ao invés de afirmar que a compreende). Em segundo lugar, a síntese (produção) requer um conhecimento linguístico maior que a análise (compreensão). Por exemplo: um falante não produz uma palavra que não conhece, enquanto é capaz de compreender, pelo contexto, o significado de uma palavra desconhecida. Em terceiro lugar, e mais importante para este trabalho, quando se aprende uma língua não materna, fenômenos como as colocações são fáceis de compreender, por sua natureza composicional (seção 1.2), mas difíceis de produzir. Este terceiro argumento é fundamental para este trabalho porque permite-nos defender que as colocações descritas em um dicionário de aprendizes (assim como em qualquer outra classe de dicionário) devem estar dispostas em um verbete de maneira tal que o seu papel como informação para produção seja evidente para o consulente.

Outro aspecto que merece destaque é a natureza funcional da TST. Em outras palavras, criou-se um modelo de representação (Modelo Sentido-Texto MST) do funcionamento de uma língua (MEL'ČUK, 1997, p. 3). Por ser um modelo representacional, o MST não está livre de equívocos. Por essa razão, os teóricos adeptos da TST estabelecem que o seu objeto de investigação é a língua em uso; o MST (através das funções lexicais, por exemplo), por sua vez, deve se adequar à mesma. Consequentemente, do ponto de vista metodológico, os estudos conduzidos pelo viés da TST partem de dados de corpora, ou seja, de manifestações da língua em uso.

Além de ser funcional, o MST é universal, ou seja, passível de ser empregado para descrever qualquer língua. Segundo Mel'čuk (ibid, p. 36), entre as suas possibilidades de aplicação estão: 1) tratamento automático da língua natural (através da elaboração de softwares de tradução automática, por exemplo); 2) aprendizagem de línguas; e 3) compilação de materiais de referência, dentre os quais se encontram os dicionários.

\subsection{A TST e a natureza tríplice do signo linguístico}

Juntamente com a concepção de língua, é fundamental compreender o conceito de signo linguístico segundo a TST. De acordo com o primeiro e o terceiro postulados gerais da TST, atribui-se um lugar de destaque na teoria para os níveis sintático e morfológico de representação da língua. Por essa razão, propõe-se que o signo linguístico está constituído de um terceiro componente, ademais do significante e do significado: a sintaxe (MEL'ČUK; MILICÉVIC (2014, p. 33-35). Este componente corresponde às propriedades combinatórias de cada signo; são imprevisíveis e, por essa razão, de natureza idiossincrática (MEL'ČUK; MILICÉVIC (ibid, p. 34). Dentre as propriedades do componente sintático de um signo estão a sua categoria morfológica do signo, as flexões (gênero, número), as irregularidades morfológicas e as colocações, as quais se constituem de "um elemento controlador que impõe a escolha de um outro signo" (ibid, p. 35).

Descrever um signo consiste, portanto, em descrever as suas propriedades. A concepção de signo proposta no marco da TST possui relação direta com o 
ensino-aprendizagem de línguas. Nesse âmbito, aprender vocabulário significa não somente aprender a relação entre significante (representação fonológica e ortográfica) e significado, mas também os padrões de combinação. Uma descrição adequada dos padrões colocacionais de um signo é importante porque, por um lado, a seleção do colocado ocorre de maneira imprevisível; é, como já citado ad supra, de caráter idiomático. Por outro lado, e no tocante ao ensinoaprendizagem de línguas não maternas, cabe observar que duas línguas podem apresentar pontos tanto de convergência como de divergência em relação a fenômenos como as colocações. Duas línguas podem selecionar colocados semelhantes para as mesmas bases correspondentes, como em "a pé", no português, e a pie, no espanhol; ou selecionar colocados diferentes para uma mesma base lexical, como em "dar trabalho" (português) e costar trabajo (espanhol); ou ainda pode haver casos em que um determinado padrão colocacional existe em uma língua, enquanto a outra expressa o mesmo sentido com uma unidade lexical apenas (ou simplesmente não o expressa).

Se se admite que existem influências entre as línguas de um falante, e que não é possível prever quando ou em que contextos a língua materna pode influenciar a língua que está sendo aprendida, um estudante brasileiro de espanhol, por exemplo, pode desempenhar-se de maneira não tão satisfatória por traduzir a base e o colocado de uma colocação em português para o espanhol sem saber se o produto da tradução realmente corresponde a uma colocação em espanhol. Somente uma descrição dos padrões colocacionais nos dicionários de aprendizes poderá fornecer informações confiáveis em relação ao funcionamento de uma língua.

O tratamento de colocações em um dicionário depende diretamente da concepção do que é uma colocação. No campo da Lexicologia, o termo colocação designa diversos conceitos conforme a perspectiva teórico-metodológica que se adota. No que concerne à TST, seus estudiosos classificam as colocações (bem como outros fenômenos de natureza combinatória) como tipos de frasemas, conforme será comentado a seguir.

\subsection{Um tipo específico de frasema: as colocações}

Além da nova concepção de signo linguístico, os estudiosos da TST defendem uma classificação de cunho lexicológico para os fenômenos de combinatória léxica que se fundamenta a partir do conceito de frasema. De acordo com Mel'čuk (2012, p. 32-33), um frasema é uma combinatória composta de duas ou mais unidades léxicas, na qual ao menos uma dessas unidades é selecionada em função da(s) outra(s) - e não por escolha própria do falante. Um frasema se exprime com determinados constituintes que não podem ser substituídos por outros (mesmo que sejam sinônimos). Por exemplo: os brasileiros exprimem o frasema intoxicação alimentar com estas (e não outras) unidades léxicas; não há liberdade para trocar o constituinte alimentar por nutricional. 
As colocações, por sua vez, são um tipo de frasema de dois constituintes que apresenta três características: é lexical, composicional e semi-restrito. Por seu viés lexicológico explicativo-combinatório, os teóricos da TST, como Polguère (2003), se contrapõem a outras perspectivas fundamentadas estritamente em critérios estatísticos, como a de Sinclair (1991) - para quem as colocações são combinatórias mais frequentes do que se poderia esperar do acaso.

Em primeiro lugar, de acordo com Mel'čuk (ibid, p. 34), possuir natureza lexical significa que o falante tem liberdade somente para determinar o sentido que deseja expressar, enquanto a seleção das unidades léxicas necessárias para expressá-lo sob a forma de um frasema (como uma colocação) é restrita ${ }^{2}$.

Em segundo lugar, a composicionalidade de um frasema está relacionada à constituição de seu sentido. Frasemas composicionais, como é o caso das colocações, são aqueles cujo sentido é produto da soma dos significados de seus constituintes $^{3}$.

Em terceiro lugar, Mel'čuk (2013, p. 138) esclarece que as colocações são semi-restritas porque um de seus constituintes (a base) "é selecionado livremente, justo por seu sentido", ao passo que o outro (o colocado) é selecionado de modo restrito, em função não apenas do sentido a ser produzido, mas também da base já pré-selecionada. No exemplo acima intoxicação alimentar, a base intoxicação é selecionada livremente; já o colocado alimentar é selecionado de maneira restrita, em função da base, para compor o sentido da colocação.

De acordo com Kahane e Polguère (2001, p. 2), por seu caráter semirestrito, as colocações estão em um ponto intermediário entre as locuções (combinatórias totalmente restritas e com sentido totalmente não composicional, como rodar a baiana), que possuem caráter idiomático, e as expressões livres (combinatórias sem restrições e composicionais). Por essa razão, as colocações são consideradas expressões semi-idiomáticas.

Além de definir o conceito de colocação por meio das propriedades citadas, a TST subdivide o fenômeno em dois tipos: colocações standard e colocações não standard. As colocações standard apresentam uma relação

\footnotetext{
${ }^{1}$ Fruto do contextualismo inglês, a abordagem de Sinclair (1991) acerca das colocações emprega análise estatística de dados de corpora para medir o quão significativa é a coocorrência de dois (ou mais) elementos [collocational strength].

${ }^{2}$ Há, por outro lado, frasemas de natureza semântico-lexical, que apresentam restrição não somente na seleção dos seus constituintes léxicos, mas também no que se refere ao sentido, em função do contexto no qual são proferidos. As fórmulas ritualísticas, tais como "bom dia", são um exemplo de restrição tanto na seleção lexical como na seleção do sentido (cf. Mel'čuk (2012, p. 34) e Mel'čuk (2013, p. 132) para mais exemplos).

${ }^{3}$ É pertinente comentar uma observação de Mel'čuk (2012, p. 39) acerca do significado do colocado. De acordo com o autor, o significado que o colocado assume na colocação nem sempre corresponde ao significado que tal UL assume livremente, ou seja, fora da colocação. A colocação furar fila é um exemplo disso, na qual o colocado furar assume um significado ('burlar') que furar não apresenta quando usado fora desse frasema.
} 
semântica entre a base e o colocado que pode ser encontrada entre diversas outras colocações (MEL' ̌́UK, 2013, p. 39-40). Já as colocações não standard apresentam, segundo Mel'čuk (ibid, p. 40) uma relação semântica entre base e colocado que surge, normalmente, em uma colocação específica (ou muito poucas).

Os diversos tipos de relação que surgem entre base e colocado são o ponto de partida para a modelização das colocações por meio de funções lexicais, originando o MST - tema da subseção a seguir.

\subsection{0 modelo sentido-texto: funções lexicais}

O MST consiste em uma proposta de modelização das relações existentes entre as unidades léxicas de uma língua para viabilizar a descrição dessas relações. As funções lexicais são

[...] aparatos formais projetados para descrever, de modo sistemático e compacto, todos os tipos de relações léxicas que ULs [i.e., unidades léxicas] de qualquer língua mantêm entre si. Essas funções almejam especificar, para qualquer UL dada, todos os correlatos léxicos que poderiam ser necessários no processo de fala (MELČUK, 2015, p. 157 - tradução nossa).

Os teóricos da TST partem da perspectiva da síntese, conforme já mencionado anteriormente. No caso de uma colocação, a seleção das unidades léxicas necessárias para expressar um sentido é determinada por restrições relacionadas ao colocado. O MST, ao propor as funções lexicais, busca descrever os tipos de relações que os diferentes colocados de uma determinada base mantêm quando são selecionados por dita base. Para evitar mal-entendidos, Mel'čuk (2015, p. 250) observa que a falta de precisão semântica das funções lexicais não permite que as mesmas sejam consideradas unidades semânticas.

As funções lexicais (portanto, as relações entre unidades léxicas) se dividem em duas classes: sintagmáticas e paradigmáticas. As primeiras tratam das combinações de unidades léxicas para predicar um sentido. As colocações, como intoxicação alimentar, são descritas por esse grupo de funções lexicais (doravante FLs). Já as segundas tratam das possibilidades de nomeação a partir de uma UL de partida; em outras palavras, são derivados semânticos.

Para fins deste trabalho, as FLs sintagmáticas são as mais pertinentes. Mel'čuk (ibid, p. 206-234) classifica-as conforme a parte do discurso a que pertencem, ou seja, de acordo com a classe gramatical a que pertence o colocado (substantivo, adjetivo, advérbio, preposição ou verbo). Em primeiro lugar, há apenas uma FL sintagmática substantiva: Centr, que denota a relação de culminância (ponto mais elevado) de uma situação. Um exemplo é a colocação

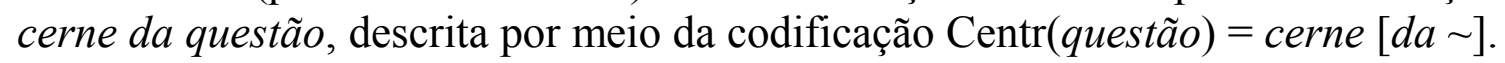

Em segundo lugar, entre as FLs sintagmáticas adjetivas e adverbiais, encontram-se Magn (maior gradação de algo, como Magn $(f o r c ̧ a)=\sim$ hercúlea), 
Plus (aumento de algo, como Plus $(l u a)=\sim$ crescente), Minus (diminuição de algo, como $\operatorname{Minus}(l u a)=\sim$ minguante), Pos (classificação positiva de algo negativo, $\operatorname{como} \operatorname{Pos}($ interferência $)=\sim$ positiva $)$, entre outros.

Em terceiro lugar, formam parte das FLs sintagmáticas preposicionais as funções $\operatorname{Loc}_{a d}($ destino, como Locad $(i r)=\sim$ para $), \operatorname{Loc}_{a d}($ partida, como Locab $(i r)=$ $\sim$ de), Instr (instrumento, como $\operatorname{Instr}(e-m a i l)=$ por $\sim$ ) e Propt (causa, como $\operatorname{Propt}($ medo $)=\sim d e)$.

Em quarto lugar, as FLs sintagmáticas verbais são as mais numerosas, envolvendo relações de suporte, causa, realização, mudanças de situação, entre outras. Algumas delas são Oper $($ fazer algo, como Oper $($ partida $)=d a r \sim)$, Caus (originar algo, como Caus(confusão) = causar $\sim$ ), Real (realização, como Real $($ juramento $)=$ prestar $\sim)$ Sympt $($ sintoma, $\operatorname{como} \operatorname{Sympt}($ fome $)=$ estar com $\sim)$.

$\mathrm{O}$ total de FLs (entre sintagmáticas e paradigmáticas) enumeradas por Mel'čuk (ibid, p. 183-234) constitui um conjunto de relações que podem ser observadas entre diversas unidades léxicas. Por essa razão, são classificadas como FLs standard (ou padrão). Por outro lado, há determinadas relações que ocorrem somente em uma UL específica, ou em uma combinatória específica. Devido ao seu caráter restrito, são classificadas como FLs não standard (ou não padrão). A relação "ter 366 dias", presente entre a base ano e o colocado bissexto, em ano bissexto, por exemplo, é não standard porque ocorre somente nessa colocação. Esse tipo de relação, entretanto, é mais rara; as FLs standard são capazes de descrever a maior parte das relações entre bases e colocados.

A descrição acurada que o MST proporciona por meio das FLs permite, por sua vez, descrever colocações de maneira mais completa em um dicionário. Uma outra vantagem do modelo é a possibilidade de descrever combinatórias léxicas em diversas línguas por meio das FLs standard.

$\mathrm{Na}$ mesma esteira de Polguère (2003), no tocante à formação de profissionais da linguagem, e do Lexique Actif du Français (MEL'ČUK; POLGUÈRE, 2007), um dicionário compilado para leigos que aplica o MST, entre tantos outros, este trabalho explora as possibilidades de aplicação das FLs para fins didáticos. Mais especificamente, propomos a descrição de colocações em dicionários de aprendizes de espanhol por meio da aplicação do MST, mas sem a sua tradicional codificação sob a forma de FLs, devido ao fato de que o público a quem se dirige essa classe de dicionários não necessariamente tem formação em Linguística.

\section{APLICAÇÃO DO MST PARA A DESCRIÇÃO DE COLOCAÇÕES}

A fim de testar nossa proposta de descrição de colocações, elegemos a UL do espanhol hambre [fome] como objeto de descrição. Segundo já comentado no início da seção 1, a TST prioriza a análise do comportamento de uma UL para a sua posterior descrição. Nossas fontes de consulta correspondem a um conjunto de dicionários de espanhol e ao Corpus del Español del Siglo XXI (CORPES, 2016). Primeiramente, analisaremos como os três dicionários de aprendizes 
existentes para o espanhol Señas (2001), DiSal (2006) e DiELE (2002) descrevem a UL hambre e se incluem colocações ${ }^{4}$. Em segundo lugar, analisaremos a descrição das colocações de hambre no seguinte conjunto de dicionários para falantes nativos de espanhol: 1) Diccionario de la Real Academia Española (DRAEe, 2014); 2) Diccionario de Uso del Español (DUE, 2001); e 3) Diccionario Combinatorio Práctico del Español Contemporáneo (DComP, 2011)5. É importante observar que, concomitante à consulta aos dicionários envolvidos nessas primeiras etapas de análise, contrastaremos a descrição de colocações oferecida por tais obras com os dados do CORPES XXI (2016).

\subsection{Descrição de colocações nos dicionários de aprendizes de espanhol}

Os únicos dicionários que apresentaram algum padrão colocacional com hambre como base são o DiELE (2002, s.v. hambre) e o Señas (2001, s.v. hambre), ao incluir hambre canina (o colocado canina tem função de intensificador) (cf. verbetes no Anexo 1).

\subsection{Descrição de colocações nos dicionários de espanhol para falantes nativos}

Em primeiro lugar, consultamos o DRAEe (2014, s.v. hambre). A única colocação presente é, novamente, hambre canina.

Em seguida, a consulta ao DUE (2001, s.v. hambre) revelou que, além de hambre canina, são incluídos conjuntos de verbos que acompanham o significante hambre quando este se manifesta sob os significados descritos nas acepções 1 e 2. Comparado com o dicionário anterior, há uma descrição mais desenvolvida dos padrões colocacionais e uma louvável tentativa de auxiliar o consulente da melhor maneira possível.

Finalmente, o DComP (2011, s.v. hambre) inclui diversos padrões de combinatória - o autor da mesma prefere não especificar a natureza da combinação entre as unidades léxicas envolvidas por meio de termos, como colocação. A maior parte dos colocados descritos pelo DUE (2001, s.v. hambre) estão presentes na obra, bem como outros mais. A obra os divide conforme a sua

\footnotetext{
${ }^{4}$ Nenhum dos três dicionários de aprendizes possui fundamentação teórica relativa à inclusão e descrição de colocações.

${ }^{5}$ Destes, o único que possui fundamentação teórica para a descrição de colocações é o DComP (2011). Seu autor, Ignacio Bosque, considera que as colocações se constituem quando uma determinada UL seleciona um conjunto de designações que compartilham um traço em comum (BOSQUE, 2001). Tal conjunto é denominado classe léxica. Quanto maior a especificidade do conteúdo de uma UL, mais restrito é o número de designações que compõe a classe léxica correspondente (e vice-versa).
} 
classe gramatical (adjetivos e verbos, no caso) e os inclui sob a forma de listas. Em alguns casos, exemplos de uso intercalam os membros das mesmas. Chama a nossa a atenção a ausência de especificação do sentido das combinações que se formam com hambre.

Utilizamos o CORPES XXI (2016) para verificar o respaldo no uso do espanhol dos padrões colocacionais presentes nos dicionários. Os resultados da combinação de cada colocado com hambre estão reunidos na tabela abaixo. Foram excluídas da análise posterior as cobinatórias que não apresentaram respaldo no corpus.

Tabela 1 - respaldo das colocações no uso do espanhol

\begin{tabular}{|c|c|c|c|c|c|c|c}
\hline colocado & $\begin{array}{c}\text { frequência } \\
\text { (registros) }\end{array}$ & $\begin{array}{c}\text { DUE } \\
\text { (2001) }\end{array}$ & $\begin{array}{c}\text { DComP } \\
\text { (2011) }\end{array}$ & $\begin{array}{c}\text { colocado } \\
\text { (requência } \\
\text { (registros) }\end{array}$ & $\begin{array}{c}\text { DUE } \\
\text { (2001) }\end{array}$ & $\begin{array}{c}\text { DComP } \\
\text { (2011) }\end{array}$ \\
\hline h. canina & 4 & $\checkmark$ & $\checkmark$ & acechar [h.] & 2 & $\times$ & $\checkmark$ \\
\hline h. voraz & 20 & $\times$ & $\checkmark$ & azotar [h.] & 2 & $\times$ & $\checkmark$ \\
\hline h. atroz & 17 & $\times$ & $\checkmark$ & aplacar [h.] & 14 & $\times$ & $\checkmark$ \\
\hline h. terrible & 5 & $\times$ & $\checkmark$ & dar [h.] & 112 & $\times$ & $\checkmark$ \\
\hline h. mortal & 0 & $\times$ & $\checkmark$ & tener [h.] & 1741 & $\checkmark$ & $\checkmark$ \\
\hline h. desmesurada & 0 & $\times$ & $\checkmark$ & pasar [h.] & 467 & $\checkmark$ & $\checkmark$ \\
\hline h. insaciable & 8 & $\times$ & $\checkmark$ & sentir [h.] & 160 & $\times$ & $\checkmark$ \\
\hline h. incontenible & 0 & $\times$ & $\checkmark$ & sufrir [h.] & 46 & $\checkmark$ & $\checkmark$ \\
\hline h. compulsiva & 0 & $\times$ & $\checkmark$ & aguantar [h.] & 60 & $\checkmark$ & $\checkmark$ \\
\hline h. acuciante & 2 & $\times$ & $\checkmark$ & saciar [h.] & 102 & $\checkmark$ & $\checkmark$ \\
\hline h. apremiante & 0 & $\times$ & $\checkmark$ & satisfacer [h.] & 21 & $\checkmark$ & $\checkmark$ \\
\hline entrar [h.] & 25 & $\times$ & $\checkmark$ & calmar [h.] & 35 & $\times$ & $\checkmark$ \\
\hline apremiar [h.] & 3 & $\times$ & $\checkmark$ & mitigar [h.] & 29 & $\times$ & $\checkmark$ \\
\hline acuciar [h.] & 1 & $\times$ & $\checkmark$ & paliar [h.] & 12 & $\times$ & $\checkmark$ \\
\hline asediar [h.] & 1 & $\times$ & $\checkmark$ & amortiguar [h.] & 4 & $\times$ & $\checkmark$ \\
\hline acosar [h.] & 8 & $\times$ & $\checkmark$ & apaciguar [h.] & 6 & $\checkmark$ & $\checkmark$ \\
\hline invadir [h.] & 2 & $\times$ & $\checkmark$ & erradicar [h.] & 18 & $\times$ & $\checkmark$ \\
\hline combatir [h.] & 34 & $\times$ & $\checkmark$ & vencer [h.] & 3 & $\times$ & $\checkmark$ \\
\hline
\end{tabular}

Fonte: elaboração própria da tabela a partir de informações disponíveis no DUE (2001), no DComP (2011) e no CORPES XXI (2016)

\section{ANÁLISE}

Aplicaram-se as FLs sintagmáticas da MST para a análise e organização das colocações de hambre. Em alguns casos, foi necessário usar FLs combinadas para descrever o sentido. Os expoentes 0,1 e 2 junto a algumas funções indicam, respectivamente, base da colocação em função de sujeito (0), presença de um sujeito externo à colocação (1) e presença de um sujeito externo e de um beneficiário externos à colocação (2). 


\subsection{Função simples magnus (Magn)}

Os colocados canina, atroz, terrible, acuciante e voraz mantêm uma relação de intensificação com a base hambre. Ao codificar essa relação por meio do MST, organizamos os colocados em ordem decrescente de frequência:

$\operatorname{Magn}($ hambre $)=\sim$ voraz $>\sim$ atroz $>\sim$ terrible $>\sim$ canina $>\sim$ acuciante.

\subsection{Função complexa antonymum verus (AntiVer)}

O colocado insaciable se relaciona com a base hambre por meio de uma negação da normalidade, pois a característica natural de "fome" é ser algo saciável. Por essa razão, juntamente com a função Ver, que indica algo que é normal a um UL, foi necessário empregar a FL paradigmática Anti, que indica justamente a negação de algo:

AntiVer(hambre $)=\sim$ insaciable.

\subsection{Função complexa functionare magnus (Func 0 Magn)}

O colocado apremiar se combina com a base hambre por meio de uma relação de manifestação ( Func $_{0}$ ) extrema (Magn). A codificação da colocação, portanto, é:

Func 0 Magn $($ hambre $)=$ apremiar .

\subsection{Função complexa incipiere functionare (IncepFunc $)_{0}$}

Os colocados entrar, despertar e invadir se combinam com a base hambre por meio de uma relação de início (Incep) da sua manifestação (Func ${ }_{0}$ ). Sua codificação é:

IncepFunc ${ }_{0}($ hambre $)=$ entrar, despertar, invadir .

\subsection{Função simples symptoma (Sympt)}

Os colocados tener, sentir e dar se combinam com a base hambre por meio de uma relação de sintoma (Sympt). Sua codificação é: 
Sympt $_{1}($ hambre $)=$ tener $\sim$, sentir $\sim$, dar $\sim$.

\subsection{Função complexa symptoma excessus (SymptExcess)}

Os colocados acuciar, asediar, acosar, azotar, pasar, sufrir e aguantar se combinam com a base por meio de uma relação de sintoma (Sympt) excessivo (Excess) - esta última, de maneira semelhante à FL adjetiva Magn, indica uma situação extrema. Sua codificação é

Sympt $_{0}($ hambre $)=$ acosar, azotar, acuciar, asediar .

Sympt $_{1}($ hambre $)=$ pasar $\sim$, sufrir $\sim$, aguantar $\sim$.

\subsection{Função complexa finire operari (FinOper)}

Os colocados saciar, satisfacer, aplacar, paliar, calmar, mitigar, amortiguar e apaciguar se combinam com a base por meio de uma relação de término (Fin) de uma atividade (Oper). Sua codificação é:

FinOper ${ }_{1}($ hambre $)=$ saciar, calmar, mitigar, satisfacer, aplacar, paliar, apaciguar, amortiguar.

\subsection{Função simples liquidare (Liqu)}

Os colocados vencer e erradicar mantêm uma relação de término definitivo (Liqu) de hambre enquanto processo de carência alimentar. Sua codificação é:

$\operatorname{Liqu}($ hambre $)=$ erradicar $\sim$, vencer $\sim$.

\subsection{Função simples híbrida labor + real (LabReal)}

O colocado combatir se combina com a base hambre por meio de uma relação de procedimento que se faz com algo (Labor). A codificação da colocação, portanto, é:

Labreal $($ hambre $)=$ combatir $\sim$. 


\section{PROPOSTA DE DESCRIÇÃO DE COLOCAÇÕES A PARTIR DO MST}

Para que se possa apresentar o produto da descrição de colocações em um dicionário de aprendizes do espanhol, é preciso adaptar a codificação de cada função, posto que, segundo sugerido por Polguère (2003) e comentado em 1.3, o público a quem se dirige essa classe de obras não necessariamente a entenderia. Nossa proposta consiste em uma adaptação da codificação por meio do emprego de uma lista de definidores empregados pelo Señas (2001) e disponível na parte final da obra. Os definidores usados para indicar as funções anteriores são:

Quadro 1 - Adaptação das FLs para a descrição de colocações

\begin{tabular}{|l|l|l|l|}
\hline \multicolumn{1}{|c|}{ FL } & \multicolumn{1}{c|}{ Adaptação } & \multicolumn{1}{c|}{ FL } & \multicolumn{1}{c|}{ Adaptação } \\
\hline Magn & [UL] extremo(a) & SymptExcess & sentir [UL] en exceso \\
\hline AntiVer & [UL] anormal & FinOper & fin de [UL] \\
\hline FuncoMagn & [UL] se revela extremo(a) & Liqu & fin definitivo de [UL] \\
\hline IncepFunco & [UL] comienza a revelarse & Labreal & hacer algo sobre [UL] \\
\hline Sympt & sentir [UL] & & \\
\hline
\end{tabular}

Fonte: elaboração própria do Quadro a partir de informações de Mel'čuk (2015) e Señas (2001)

Conforme já comentado na seção 1 , as colocações oferecem mais dificuldade em relação à sua produção, pois, quando se trata de uma língua não materna, os colocados que acompanham uma determinada base não são óbvios (especialmente quando há divergência entre os colocados selecionados por duas línguas). Ademais, e dado o anisomorfismo existente entre as línguas, um mesmo sentido pode estar veiculado por uma UL em uma língua e por uma colocação na outra. Por esses dois motivos, e ante o dever de auxiliar o consulente em tarefas de síntese (produção), todas as adaptações funcionarão como distinguidores semânticos para guiar do sentido às colocações que o expressam. A seguir, apresentamos uma amostra do espaço destinado à descrição de colocações em um dicionário de aprendizes do espanhol: 
Quadro 2 - esboço do segmento informativo referente aos padrões colocacionais

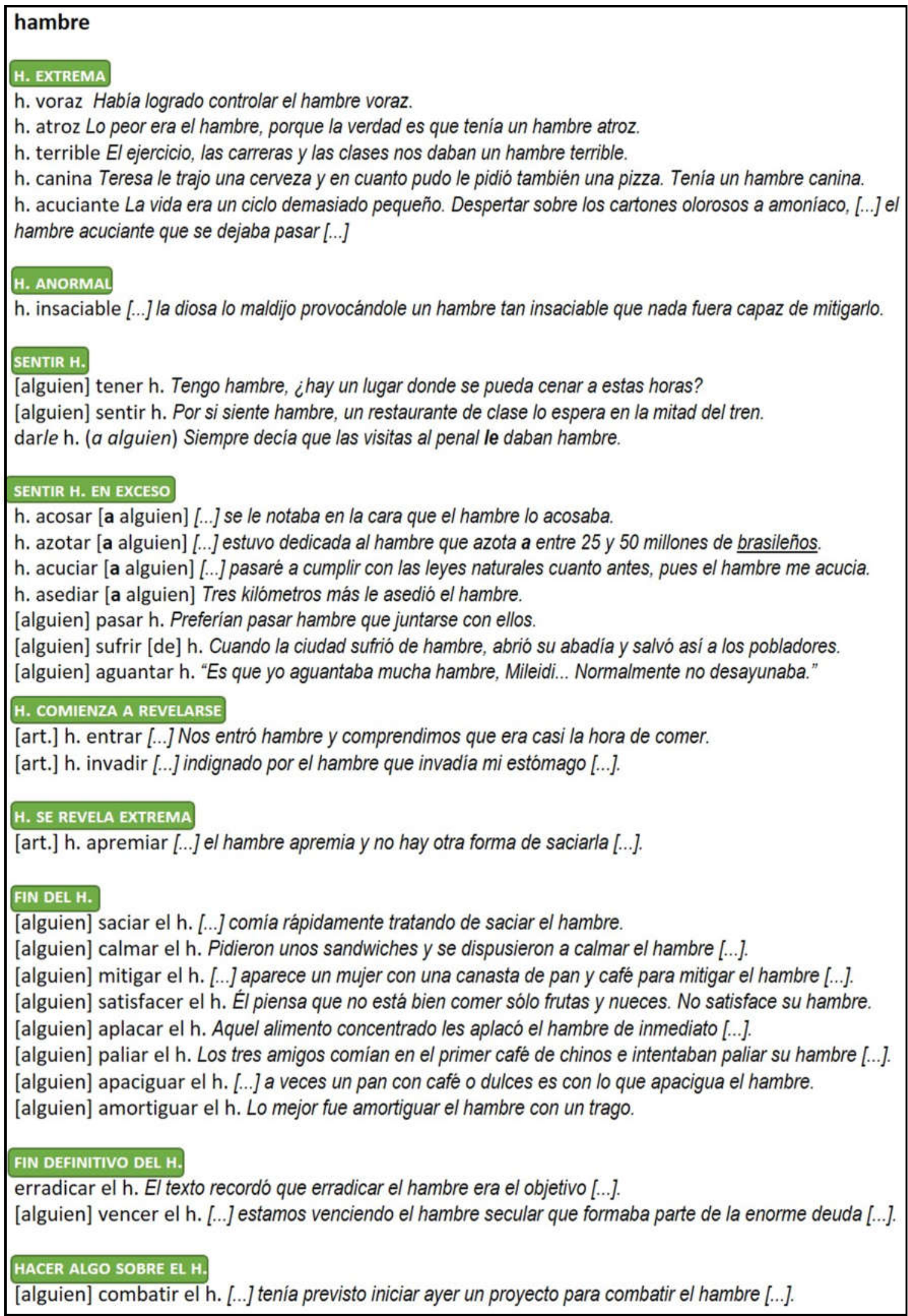

Fonte: elaboração própria. Os exemplos foram extraídos do CORPES XXI (2016) 
Os distinguidores semânticos estão marcados tipograficamente em fundo verde. Os exponentes referentes aos actantes envolvidos nos padrões colocacionais foram indicados por meio de marcações de sujeito ([alguien]), objeto direto de pessoa ([a alguien $]$ ) e beneficiário ([a alguien $]$ ). $\mathrm{O}$ consulente poderia beneficiar-se ainda com o acréscimo de uma abonação (exemplo) para ilustrar o comportamento de cada colocação ${ }^{6}$.

\section{CONSIDERAÇÕES FINAIS}

O exemplo de aplicação exposto ofereceu um quadro descritivo dos padrões combinatórios da unidade léxica hambre à luz dos pressupostos teóricos da TST. A nossa experiência revela que essa metodologia demonstra ser muito útil para fins de descrição lexicográfica. Embora o presente trabalho se baseia em um único fato de língua, acreditamos que a TST tem muito a oferecer para a práxis lexicográfica, pois a sua metodologia elenca fatos de combinatória léxica idiossincráticos do espanhol. Trata-se de um aspecto da língua em uso a cuja descrição o aprendiz precisa ter acesso para que possa valer-se de um auxílio na produção de textos que tenham o “jeito” do espanhol.

\section{REFERÊNCIAS}

ANAIS DO IX SEMINÁRIO DE ENSINO DE LÍNGUAS ESTRANGEIRAS (SELES). Vol. 2. Passo Fundo: Editora UPF, 2017b. p. 442-459. Disponível em: $<$ http://selesselm.upf.br/images/pdf/anais_seles_e selm_PARTE2.pdf $>$ Acesso em 30 jan. 2018

BORBA, laura Campos de. Panorama da Lexicografia Hispânica: subsídios para o professor de ELE. Saarbrücken: NEA - Novas Edições Acadêmicas, 2017 a.

. "Dicionários para aprendizes na lexicografia hispânica: señas (2001)", Salamanca (1996) e Espasa Calpe (2002). In: STURM, Luciane; VALÉRIO, Patrícia. (orgs.).

BOSQUE, Ignacio. "Sobre el concepto de colocación y sus limites". In: Lingüística Española Actual, Madrid, v. 23, n. 1, 2001, p. 9-40.

CORPES XXI. REAL ACADEMIA ESPAÑOLA. In: Corpus del Español del Siglo XXI. 2016. Disponível em: <http://www.rae.es/recursos/banco-de-datos/corpes-xxi> Acesso em: 25 fev. 2018

\footnotetext{
${ }^{6}$ Quanto ao layout do verbete, por questões de espaço no presente trabalho, foi necessário dividi-lo em duas colunas. Para um dicionário impresso, propõe-se que haja apenas uma; já um dicionário eletrônico poderia exibir os padrões a partir de um comando presente em cada distinguidor.
} 
DComP. BOSQUE, Ignacio. Diccionario combinatorio práctico del español contemporáneo. Madrid: SM, 2011.

DiELE. ESPASA CALPE. Diccionario de la Lengua Española para estudiantes de Español. Madrid: Espasa Calpe, 2002.

DiSal. GUTIÉRREZ CUADRADO, Juan. Diccionario Salamanca de la Lengua Española. Madrid: Santillana, 2006.

DRAEe. REAL ACADEMIA ESPAÑOLA. Diccionario de la Real Academia Española. $23^{\mathrm{a}}$ edición. Disponível em: $<$ http://dle.rae.es/?id=DgIqVCc $>$ Acesso em: 28 fev. 2018

DUE, MOLINER, María. Diccionario de uso del español. Madrid: Gredos, 2001.

KAHANE, Sylvain; POLGUÈRE, Alain. "Un langage formel d'encodage des fonctions lexicales et son application à la modélisation des collocations". In: DAILLE, B.; WILLIAMS, G. La Collocation - Journée d'études de l'ATALA. Paris, 2001. p. 15-18.

MEL'CUK, Igor. Vers une linguistique Sens-Texte. Leçon inaugurale. Paris: Collège de France, 1997.

. "Phraseology in the language, in the dictionary and in the computer". In: KUIPER, Koenraad. (ed.). Yearbook of Phraseology. v. 3. Berlin: De Gruyter, 2012. p. 31-56.

. Les collocations. Cahiers de Lexicologie, v. 102, 2013, p. 129-149.

2015.

. Semantics: from meaning to text. Vol. 3. Philadelphia: John Benjamins,

MEL'CUK, Igor; MILICÉVIC, Jasmina. Introduction à la linguistique. Vol. 1. Paris: Hermann Éditeurs, 2014.

MEL'CUK, Igor; POLGUÈRE, Alain. Lexique actif du français. Bruxelles : De Boeck, 2007.

PCIC. INSTITUTO CERVANTES. Plan Curricular del Instituto Cervantes. Niveles de referencia para el español. Madrid: Biblioteca Nueva, 2006.

. "Collocations et fonctions lexicales : pour un modèle d'apprentissage". In : GROSSMANN, Francis; TUTIN, Agnès. (dir.). Les Collocations. Analyse et traitement, collection Travaux et Recherches en Linguistique Appliquée. Amsterdam: De Werelt, 2003, p. 117-133.

SEÑAS. UNIVERSIDAD ALCALÁ DE HENARES. Señas - Diccionario para la Enseñanza de la Lengua Española para Brasileños. São Paulo: Martins Fontes, 2001. 
SINCLAIR, John. Corpus, Concordance and Collocation. Oxford: Oxford University Press, 1991.

Laura Campos de Borba LauraCBorba@hotmail.com

Recebido em: 6 mar. 2018 Aceito em: 14 jun. 2018

Publicado em: 19 ago. 2018 